Original article

\title{
Abnormal brain oscillations persist after recovery from bipolar depression
}

\author{
P. Canali ${ }^{\text {a,* }}$, S. Casarotto ${ }^{\text {b }}$, M. Rosanova ${ }^{\text {b,e }}$, G. Sferrazza-Papa ${ }^{\text {a }}$, A.G. Casali ${ }^{f}$, O. Gosseries $^{\text {c,d }}{ }^{\text {, }}$ \\ M. Massimini ${ }^{\text {b }}$, E. Smeraldi ${ }^{\text {a }}$, C. Colombo ${ }^{\text {a }}$, F. Benedetti ${ }^{\text {a }}$ \\ ${ }^{a}$ Department of clinical neurosciences, scientific institute Ospedale San Raffaele, university Vita-Salute San Raffaele, San Raffaele Turro, 20, via Stamira \\ d'Ancona, 20127 Milano, Italy \\ bepartment of biomedical and clinical sciences “L. Sacco”, università degli Studi di Milano, Milano, Italy \\ ${ }^{\mathrm{c}}$ Coma science group, GIGA research \& neurology department, university hospital of Liège, Liège, Belgium \\ ${ }^{\mathrm{d}}$ Center for sleep and consciousness, Postle laboratory, department of psychology and psychiatry, university of Wisconsin, Madison, WI, USA \\ ${ }^{\mathrm{e}}$ Fondazione Europea di Ricerca Biomedica, FERB Onlus, Milan, Italy \\ ${ }^{\mathrm{f}}$ Institute of science and technology, Federal university of São Paulo, 330, Rua Talim, São José dos Campos, Brazil
}

\section{A R T I C L E I N F O}

\section{Article history:}

Received 19 July 2016

Received in revised form 15 October 2016

Accepted 16 October 2016

Available online 3 February 2017

\section{Keywords:}

TMS/EEG

Gamma oscillations

Biomarkers

Bipolar disorder

GABAergic circuits

\begin{abstract}
A B S T R A C T
When directly perturbed in healthy subjects, premotor cortical areas generate electrical oscillations in the beta range $(20-40 \mathrm{~Hz})$. In schizophrenia, major depressive disorder and bipolar disorder (BD), these oscillations are markedly reduced, in terms of amplitude and frequency. However, it still remains unclear whether these abnormalities can be modulated over time, or if they can be still observed after treatment. Here, we employed transcranial magnetic stimulation (TMS) combined with EEG to assess the frontal oscillatory activity in eighteen BD patients before/after antidepressant treatments (sleep deprivation and light therapy), relative to nine healthy controls. In order to detect dominant frequencies, event related spectral perturbations (ERSP) were computed for each TMS/EEG session in all participants, using wavelet decomposition. The natural frequency at which the cortical circuit oscillates was calculated as the frequency value with the largest power across $300 \mathrm{~ms}$ post-stimulus time interval. Severity of depression markedly decreased after treatment with 12 patients achieving response and nine patients achieving remission. TMS/EEG resulted in a significant activation of the beta/gamma band response (21$50 \mathrm{~Hz}$ ) in healthy controls. In patients, the main frequencies of premotor EEG responses to TMS did not significantly change before/after treatment and were always significantly lower than those of controls $(11-27 \mathrm{~Hz})$ and comparable in patients achieving remission and in those not responding to treatment. These results suggest that the reduction of natural frequencies is a trait marker of $\mathrm{BD}$, independent from the clinical status of the patients. The present findings shed light on the neurobiological underpinning of severe psychiatric disorders and demonstrate that TMS/EEG represents a unique tool to develop biomarkers in psychiatry.
\end{abstract}

(c) 2016 Elsevier Masson SAS. All rights reserved.

\section{Introduction}

Currently, bipolar disorder (BD) is the sixth leading cause of disability [1,2] and affects nearly $1-2 \%$ of the population worldwide [3]. During illness episodes of BD patients experience pervasive changes in mood and cognition, and deficits in executive functions, attention, psychomotor speed, verbal and visual memory often persist in euthymia [4], suggesting persistent changes in brain structure and function [5]. Identifying trait

\footnotetext{
* Corresponding author. Tel.: +39 02 26433156; fax: +3902 26433265 .

E-mail address: canali.paola@gmail.com (P. Canali).
}

markers of persistently abnormal brain function is then a priority to identify new targets for treatment of these dysfunctions [6].

High frequency brain oscillations are rhythmic electrical phenomena, which are generated spontaneously and in response to stimuli, and which parallels the natural mechanism for carrying neural information among brain areas [7] and integrating cortical modules [8]. They are modified in many neuropsychiatric conditions, and in cognitive impairment [9]. Accordingly, they are also markedly reduced in $\mathrm{BD}$. Cross-sectional studies suggest that alterations in the GABA/glutamatergic systems, and in neural circuits that regulate cognitive processing, may be reflected through in altered brain oscillations in BD [10]: even in euthymic conditions, patients showed reduced gamma oscillations $[11,12]$, 
reduced long distance gamma coherence between frontal and temporoparietal regions [11], and decreased beta synchronization in the frontal region [13].

The combination of transcranial magnetic stimulation with electroencephalogram (TMS/EEG) represents a non-invasive, perturbational approach to precisely identify the integrity of thalamocortical circuits by directly challenging the brain's capacity to produce and sustain oscillatory activity [14-16]. With TMS/EEG, we previously reported that each cortical region tends to oscillate at a specific natural frequency [17], and that the main frequencies of frontal EEG responses to TMS were significantly reduced in patients with $\mathrm{BD}$, major depressive disorder, and schizophrenia relative to healthy subjects (11-27 Hz vs $21-50 \mathrm{~Hz}$, respectively) [18].

It remains unclear if these abnormalities change over time, and no longitudinal study has yet assessed high-frequency oscillations before and after response to antidepressants. Sleep deprivation and light therapy (SD + LT) provide a model of antidepressant treatment which allows to study the biological correlates of psychopathology at close time points and without the confounding effects of drugs [19]. Using this model antidepressant, we previously showed that response associates both, with TMS/EEG evoked measures of cortical excitability [20], with cortical volumes and function, and concentrations of neurotrophins [21]. Here, we aimed at investigating the oscillatory properties of the frontal cortex by TMS/EEG before and after treatment with combined chronotherapeutic techniques (SD + LT).

\section{Materials and methods}

\subsection{Participants, treatment and data collection}

We studied 18 consecutively admitted inpatients ( 14 females; mean \pm SD age: $42.6 \pm 9.6$; age at onset of illness: $27.9 \pm 7.4$; years at school: $13.5 \pm 4.3$; previous depressive episodes: $6.1 \pm 5.3$; previous manic episodes: $3.1 \pm 2.2$ ) suffering from a major depressive episode, without psychotic features, affected by BD (DSM-IV criteria, SCID interview). Inclusion criteria were a baseline Hamilton depression rating scale (HDRS) score of 18 or higher; absence of other diagnosis on axis I and of mental retardation on axis II; absence of pregnancy, history of epilepsy, or major medical and neurologic disorders; absence of a history of drug or alcohol dependence within the last 6 months; no treatment with long-acting neuroleptic drugs in the last 3 months before admission. Nine healthy participants ( 6 females, age $38.9 \pm 10.5)$ served as controls. After a complete description of the study, a written informed consent was obtained. All the research activities were approved by the local ethical committee.

All patients were treated for one week with SD + LT [22]. They were totally sleep deprived on days 1,3 and 5, from 7:00 am to 7:00 pm of the subsequent day; and were allowed to recover sleep on days 2, 4, and 6 . All patients were administered a 10,000-lux white light for 30 minutes, given at 3:00 am during the SD night and in the morning after recovery sleep, half an hour after awakening. Five patients were on ongoing lithium treatment (mean \pm SD daily dose: $750 \pm 251 \mathrm{mg}$ ), and continued it; thirteen started it together with the chronotherapeutic procedure $(600 \mathrm{mg} /$ day) to enhance its effect and prevent relapse [22]. No other psychotropic drug was administered during the study.

Severity of depression was rated at baseline (day 0 ) and after treatment (day 7) on the 21-item HDRS.

\section{2. $T M S / E E G$ procedure}

TMS/EEG was performed before and after treatment (day 0 and 5 , at 08:30 am). Stimulation parameters (location, intensity, angle, coil orientation) were maintained constant and reproducible through a neuronavigation system (Nexstim, Helsinki, Finland).
Spontaneous EEG was continuously recorded for about 3 min before each TMS/EEG recording session.

Prior to the TMS/EEG recording sessions, anatomical whole head images of each patient were obtained with a 3.0-T scanner (Gyroscan Intera, Philips, Netherlands; T1-weighted MPRAGE sequence; TR $2500 \mathrm{~ms}$, TE $4.6 \mathrm{~ms}$, yielding 220 transversal slices with a thickness of $0.8 \mathrm{~mm}$ ). The acquired volume was then segmented to obtain a 3D model of the surface of the scalp and of the cortex, to be uploaded in the brain navigation software.

The experimental setup included TMS with a Focal Bipulse 8-Coil (Eximia TMS stimulator; Nexstim Ltd., Helsinki, Finland) equipped with a navigated brain stimulation system (NBS; Nexstim Ltd.) and a 3D-infrared tracking position sensor unit (Polaris, Northem Digital Inc., Waterloo, Canada). EEG was recorded with a 60-channel TMScompatible EEG amplifier (Nexstim Ltd, Helsinki, Finland) equipped with sample-and-hold circuits that prevent the recording from the powerful TMS-related artifacts [23]. EEG cap was repositioned before each session, controlling for reproducibility of location using the NBS system. Impedances were kept below $5 \mathrm{k} \Omega$. EEG signals were band-pass filtered between $0.1-500 \mathrm{~Hz}$, and sampled at $1.450 \mathrm{~Hz}$ with 16-bit resolution. Electro-oculogram was recorded with two additional electrodes on the forehead to measure ocular movements and blinks.

This equipment provides in real time the TMS coil position and subject's head, within the reference space of individual magnetic resonance imaging (MRI) by the co-registration between the fiducials points (nasion, left tragus and right tragus) selected on the individual MRI with the corresponding digitized scalp landmarks. The exact location of the stimulation site was adjusted on the individual MRI in order to prevent accidental muscle twitches that often affect EEG recordings, and to estimate the electrical field induced by TMS pulses, which depends on the stimulation intensity $(\mathrm{V} / \mathrm{m})$. The TMS intensity was adjusted according to the maximum electric field intensity (expressed in $\mathrm{V} / \mathrm{m}$ ) estimated on the cortical surface, rather than relying on individual motor threshold or on the percentage of maximum stimulator output.

To ensure significant EEG responses [24] TMS intensity was always $>90 \mathrm{~V} / \mathrm{m}$ as estimated by the NBS system, for each patient. TMS was delivered on the convexity of the middle caudal portion of the superior frontal gyrus close to the midline (Brodmann's areas 6), with the current perpendicular to its main axis. These brain areas showed the highest changes of metabolic rate and EEG correlates between wake and sleep [25] and have been associated with the antidepressant effects of SD [19].

To obtain significant TMS evoked potentials (TEP) with a good signal-to-noise ratio, about 200-300 stimuli were delivered for each session at frequency randomly distributed between $1.5-1.8 \mathrm{~s}$ (equivalent to about $0.5-0.6 \mathrm{~Hz}$ ). This stimulation rate does not induce significant reorganization/plasticity processes that might possibly interfere with longitudinal measurements [26]. During TMS stimulation patients were laying on an ergonomic chair, with eyes open looking at a fixation point on a screen, and wore inserted earplugs continuously playing a masking noise that abolished the auditory potentials elicited by TMS-associated click [27].

\subsection{Data analysis}

Data analysis was carried out using Matlab (2007b, The Mathworks Inc., Natick, MA). TMS evoked potentials containing activity from sources other than neural, such as spontaneous muscles activity or ocular movements, were automatically identified and rejected using a semi-automatic algorithm (EOG $>70 \mu \mathrm{V}$ or absolute power of EEG channel F8 above $25 \mathrm{~Hz},>0.9 \mu \mathrm{V}^{2}$ ) [24]. Thereafter, single trials and channels contaminated by residual artifacts were visually inspected and excluded from further analysis. Selected trials were band-pass 
filtered between $2-80 \mathrm{~Hz}$, down-sampled to $725 \mathrm{~Hz}$, and rereferenced to the common average reference. Each TMS-evoked response was obtained by averaging 150-250 artifact free trials.

In order to quantify the responses in the time-frequency domain [28], from each TMS/EEG session, we measured the eventrelated spectral perturbation (ERSP) changes in the power spectrum using wavelet decomposition (3.5 oscillation cycles) across single-trials at the channel closest to the stimulation site (selected channel for single subject: Fz $n=1$; FC1 $n=8$; FCz $n=8$; FC2 $n=4 ; \mathrm{C} 1 n=1 ; \mathrm{Cz} n=4 ; \mathrm{C} 2 n=1$ ). The ERSP was normalized by subtracting the mean baseline power spectrum. Significant ERSP were evaluated by applying a bootstrap statistical method based on a surrogate distribution randomly derived from the prestimulus onset $(-700-50 \mathrm{~ms})$. Statistical significance level was set at $P<0.01$ and only significant values were considered in the analysis. Averaged ERSP values across all trials of a session were calculated between 8 and $50 \mathrm{~Hz}$ ( $1 \mathrm{~Hz}$ bin resolution) over a 20-300 millisecond time window, corresponding to the main EEG activity evoked by TMS. The natural frequency was computed as the frequency bin with the largest cumulated ERSP over time [17].
Data were analyzed with Student's t-test and Pearson's correlation. Moreover, we performed a repeated measures ANOVA on TMS evoked natural frequencies before/after treatment, with time and response to treatment as independent factors. Analyses were performed in the context of the general linear model (GLM) $[29,30]$. The significance of the effect of the single independent factor on each dependent variable was estimated (least squares method) by parametric estimates of predictor variables and following standard computational procedures [31].

\section{Results}

Severity of depression markedly decreased after treatment (HDRS day 0: $23.2 \pm 5.8$; day $7: 8.5 \pm 7.5 ; t=8.85, P<0.00001$ ), with 12 patients (66.6\%) achieving response (HDRS 50\% reduction) and 9 patients $(50 \%)$ achieving remission (HDRS $<8$ ).

Data obtained with the TMS/EEG procedure are showed in Fig. 1. Top panel shows the average EEG responses to TMS (grey traces) for the channel closest to the stimulation site (black trace) over the premotor area. To detect the natural frequency, we measured the event-related spectral perturbation (ERSP) for each

\section{Healthy Control}

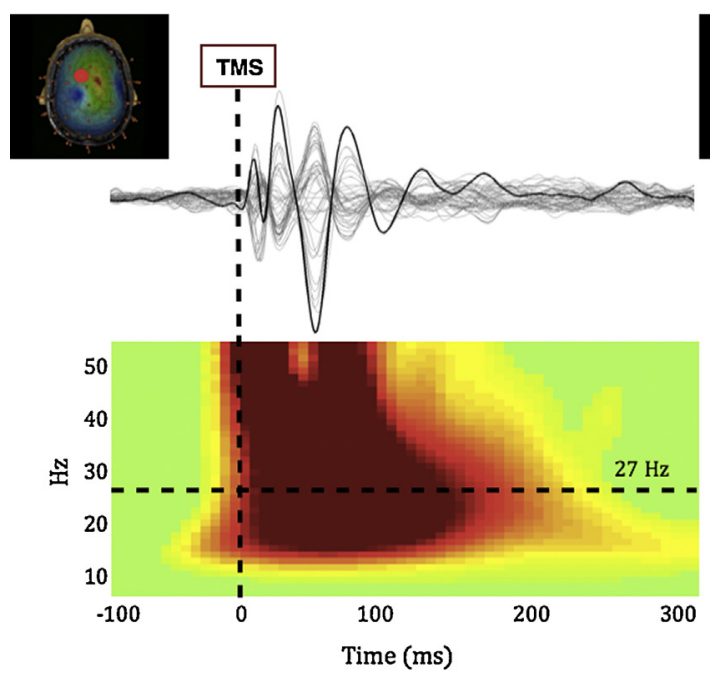

Bipolar Disorder

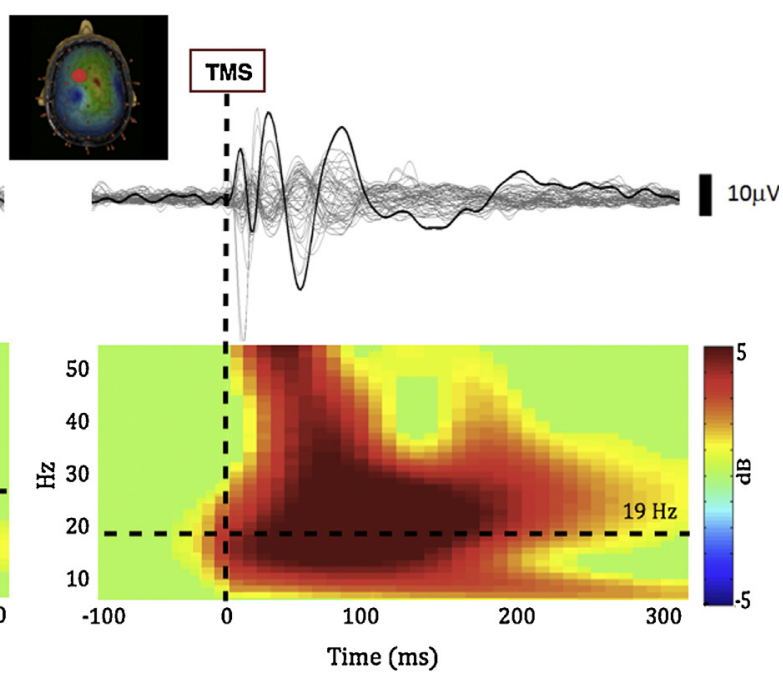

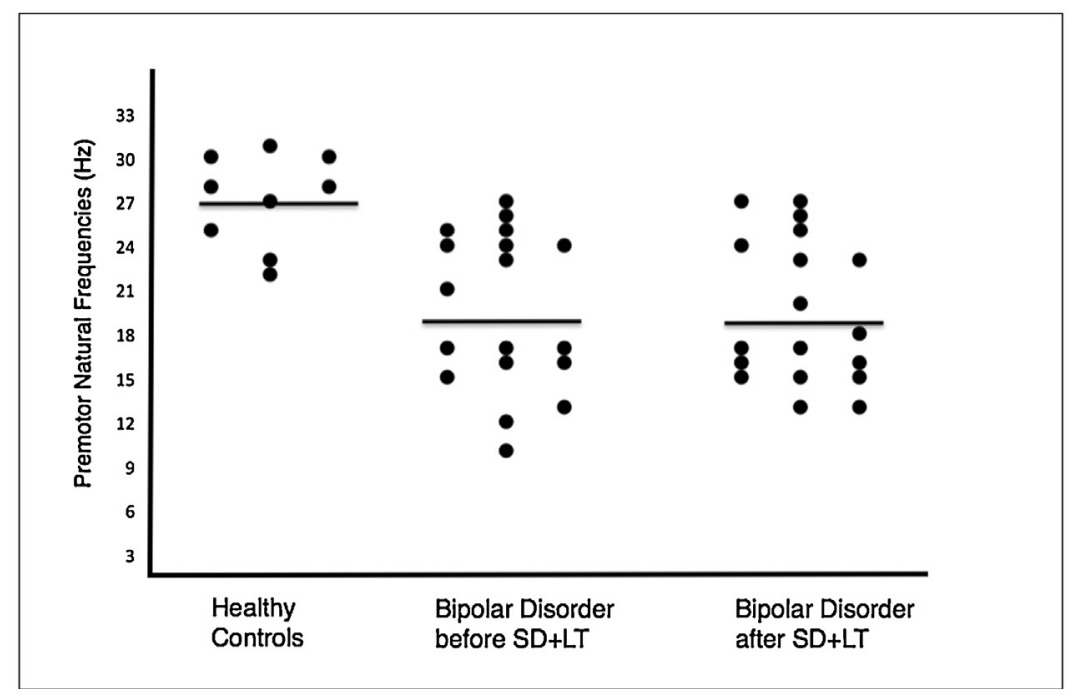

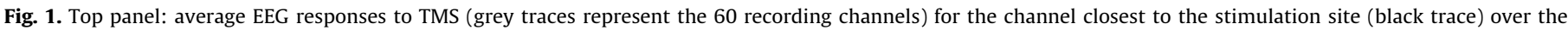

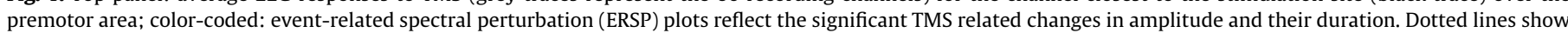

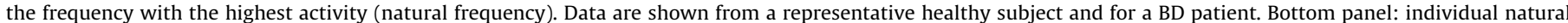
frequency values for healthy control subjects and patients with bipolar disorder before/after the antidepressant treatments (SD + LT). 
single subject. Specifically, we cumulated the ERSP between $8-50 \mathrm{~Hz}$ and $20-300 \mathrm{~ms}$ evoked by TMS. The main frequency at which a system oscillates was selected by the frequency showing the largest activity across time. Data shown in Fig. 1 are from a representative subject.

TMS resulted in a significant activation of the beta/gamma band response in healthy controls $(27.0 \pm 3.0 \mathrm{~Hz}$, range 23-33). In patients, the main frequencies of frontal EEG responses to TMS did not significantly change before/after treatment (day 0: $19.44 \pm 5.41 \mathrm{~Hz}$, range $10-27$; day $7: 19.30 \pm 4.99 \mathrm{~Hz}$, range $13-$ $27 ; t=0.391, P=0.700$ ), and at both time points were significantly lower than those of controls (day $0: t=3.87, P=0.0006$; day 7: $t=4.22, P=0.0002$ ). Values were closely similar in patients eventually achieving remission, or not (remitters: day 0, $20.37 \pm 5.50$ and day $7,20.21 \pm 4.93$; non remitters: day $0,18.51 \pm 5.46$ and day 7 , $18.40 \pm 5.18)$. Values before/after treatment were highly correlated $(r=0.9616, P<0.0001)$.

A repeated measures ANOVA gave no effects of time and of response to treatment on EEG measures before/after treatment (time: $\mathrm{F}_{1,16}=0.14, P=0.709$; response: $\mathrm{F}_{1,16}=0.56, P=0.467$; interaction: $\left.F_{1,16}=0.006, P=0.941\right)$. Moreover, natural frequencies did correlate neither with HDRS scores before/after treatment, nor with their delta change, nor with clinical and demographic characteristics of the patients.

\section{Discussion}

In this study, we assessed natural frequencies of cortical circuits before and after antidepressant treatment in BD. Notably, neither changes over time nor any correlation with the severity of depression were observed. Evoked brain oscillations remained lower than those of healthy controls, and comparable in patients achieving remission and in those not responding to treatment. This suggests that the reduction of natural frequencies is a trait marker of $\mathrm{BD}$, independent from the current clinical status of the patients.

The present observation that successful antidepressant treatment is unable to normalize thalamocortical circuits in BD is consistent with previously reported reduced gamma coherence in euthymic patients with BD [11]. Recently, we showed that the reduction of natural frequencies is detectable in schizophrenia, BD, and major depressive disorder [18]. The observation of its persistence in BD after treatment now suggests that this trait marker could be linked to persistent trait characteristics of brain structure and function in BD. Based on existing literature, the most likely candidates are GABAergic and glutamatergic neurotransmission.

Fast activity of parvalbumin GABA interneurons is needed for high-frequency oscillations [32,33], with gamma suppression resulting from their inhibition [34]. GABA inhibitory interneurons produce and sustain complex large-scale network oscillations in fast frequency bands (40-100 Hz) [35], also generating inhibitory potentials in excitatory pyramidal neurons [36]. This GABAergic activity results in gamma oscillations [37-40], and is reduced in BD. Post-mortem studies showed a reduced density of GABAergic neurons in the cortex of patients with $\mathrm{BD}$ [35], with a reduction in the numerical density of parvalbumin- and somatostatin-positive interneurons [41] and in the prefrontal cortex reduced markers of the parvalbumin subpopulation [42]. In vivo, MR spectroscopy confirmed low GABA levels in the prefrontal cortex [43].

In the generation and maintenance of high frequency oscillations, GABAergic mechanisms are likely to interact with glutamatergic mechanisms, involving NMDA and AMPA receptors [44]. Glutamate levels in brain tissue were increased both in vivo [45], and post-mortem [46] in patients with BD. Genetic ablation of NMDA receptors in parvalbumin interneurons resulted in increased gamma power [47]. The decrease of excitatory input to fast spiking parvalbumin interneurons, induced by the NMDA antagonist ketamine $[48,49]$, lead to increased gamma activity [50-52]. AMPA receptor antagonists inhibit gamma oscillations [32]. Ketamine has rapid and marked antidepressant effects in BD [53], and it is not the only therapeutic agent to act on brain oscillations. Evoked beta responses from lithium-treated BD patients were higher than those in both drug-free euthymic BD patients and healthy controls [54], and interestingly, post-mortem GABA levels were increased after lithium treatment [46].

Altogether, these data support the hypothesis that abnormal GABAergic and glutamatergic neurotransmission could be critical to explain the abnormal gamma oscillations observed in our patients [34,55], and that these abnormalities are persistent and detected both during the lifespan, and post-mortem. Alternative interpretations cannot be ruled out; for instance, EEG power in high frequency bands has been positively correlated with arterial spin labelling MRI measures of resting cerebral perfusion in healthy subjects [56].

Fast oscillatory dynamics are needed for large-scale integration and synchronous communication between brain regions, are thought to parallel the emergence of coherent behavior and cognition [57], and are implicated in many brain functions including the processing of sensory stimuli [36], language comprehension [58], cognitive skills [59], cognitive processing in recognition memory [60]. It is tempting to hypothesize that their persistent abnormality in BD could mark the persistent deficits in higher cognitive functions and brain network connectivity associated with the disorder $[4,5,61]$. Further research is needed to clarify this issue.

Strengths of the present study include a focused research question, state-of-the-art TMS/EEG methods, and straightforward effects. However, our experimental setting did not allow to directly assess the role of deeper structures, such as hippocampus, which contribute to gamma oscillations [62]. We obtained an excellent power to study group differences, but could not consider other biological markers, gene variants, and their interaction with clinical variables. Patients were non drug-naïve. Recruitment was in a single ethnic group, thus raising the possibility of population stratifications limiting the generalizability of the findings.

\section{Authors and contributors}

F.B., P.C., M.M. and M.R. designed the study. E.S., F.B. and M.M. obtained the funding. C.C., F.B. and P.C. were involved in participants' recruitment and selection. P.C. and G.S.P. collected the clinical data with the supervision of F.B. P.C., S.C., G.S.P. and O.G. carried out the TMS-EEG experimental procedures. P.C., M.R., M.M., S.C., G.S.P., A.C. and OG designed the data analyses and carried it out with contributions from F.B. P.C. and F.B. wrote the first draft of the manuscript, with other authors contributing to data interpretation and final manuscript preparation. F.B. and P.C. had full access to all of the data in the study and take responsibility for the integrity of the data and the accuracy of the data analysis. All authors take final responsibility for the decision to submit for publication.

\section{Funding}

The authors declare no funding source for the present work.

\section{Disclosure of interest}

The authors declare that they have no competing interest.

\section{Acknowledgments}

None. 


\section{References}

[1] Bauer MS, Kirk GF, Gavin C, Williford WO. Determinants of functional outcome and healthcare costs in bipolar disorder: a high-intensity follow-up study. J Affect Disord 2001;65:231-41.

[2] Murray CJ, Lopez AD. Evidence-based health policy - lessons from the Global burden of disease study. Science 1996;274:740-3.

[3] Ketter TA. Nosology, diagnostic challenges, and unmet needs in managing bipolar disorder. J Clin Psychiatry 2010;71:e27.

[4] Poletti S, Sferrazza Papa G, Locatelli C, Colombo C, Benedetti F. Neuropsychological deficits in bipolar depression persist after successful antidepressant treatment. J Affect Disord 2014;156:144-9.

[5] Poletti S, Bollettini I, Mazza E, Locatelli C, Radaelli D, Vai B, et al. Cognitive performances associate with measures of white matter integrity in bipolar disorder. J Affect Disord 2015;174:342-52.

[6] Biomarkers Definitions Working G. Biomarkers and surrogate endpoints: preferred definitions and conceptual framework. Clin Pharmacol Ther 2001;69:89-95.

[7] Buzsaki G, Draguhn A. Neuronal oscillations in cortical networks. Science 2004;304:1926-9.

[8] Nikolic D, Fries P, Singer W. Gamma oscillations: precise temporal coordination without a metronome. Trends Cogn Sci 2013;17:54-5.

[9] Başar E. Brain oscillations in neuropsychiatric disease. Dialogues Clin Neurosci 2013; $15: 291-300$.

[10] Ozerdem A, Kocaaslan S, Tunca Z, Basar E. Event related oscillations in euthymic patients with bipolar disorder. Neurosci Lett 2008;444:5-10.

[11] Ozerdem A, Guntekin B, Atagun I, Turp B, Basar E. Reduced long distance gamma $(28-48 \mathrm{~Hz})$ coherence in euthymic patients with bipolar disorder. J Affect Disord 2011;132:325-32.

[12] Shaw A, Brealy J, Richardson H, Muthukumaraswamy SD, Edden RA, John Evans C, et al. Marked reductions in visual evoked responses but not gammaaminobutyric acid concentrations or gamma-band measures in remitted depression. Biol Psychiatry 2013;73:691-8.

[13] Chen SS, Tu PC, Su TP, Hsieh JC, Lin YC, Chen LF. Impaired frontal synchronization of spontaneous magnetoencephalographic activity in patients with bipolar disorder. Neurosci Lett 2008;445:174-8.

[14] Buzsaki G, Watson BO. Brain rhythms and neural syntax: implications for efficient coding of cognitive content and neuropsychiatric disease. Dialogues Clin Neurosci 2012;14:345-67.

[15] Canali P. A role for TMS/EEG in neuropsychiatric disorders. Neurol Psychiatry Brain Res 2014;20:37-40.

[16] Rosanova M, Casarotto S, Pigorini A, Canali P, Casali AG, Massimini M. Combining transcranial magnetic stimulation with electroencephalography to study human cortical excitability and effective connectivity. In: Fellin T, Halassa M, editors. Neuronal network analysis concepts and experimental approaches. New York, Heidelberg: Springer; 2012. p. 435-57.

[17] Rosanova M, Casali A, Bellina V, Resta F, Mariotti M, Massimini M. Natural frequencies of human corticothalamic circuits. J Neurosci 2009;29:7679-85.

[18] Canali P, Sarasso S, Rosanova M, Casarotto S, Sferrazza-Papa G, Gosseries O, et al. Shared reduction of oscillatory natural frequencies in bipolar disorder, major depressive disorder and schizophrenia. J Affect Disord 2015;184:111-5.

[19] Benedetti F, Bernasconi A, Blasi V, Cadioli M, Colombo C, Falini A, et al. Neural and genetic correlates of antidepressant response to sleep deprivation - A functional magnetic resonance imaging study of moral valence decision, in bipolar depression. Arch Gen Psychiatry 2007;64:179-87.

[20] Canali P, Sferrazza Papa G, Casali AG, Fecchio M, Pigorini A, Schiena G, et al. Changes of cortical excitability as biomarkers of antidepressant response in bipolar depression. Bipolar Disord 2014;16:809-19.

[21] Benedetti F, Poletti S, Hoogenboezem TA, Locatelli C, Ambree O, de Wit H, et al. Stem cell factor (SCF) is a putative biomarker of antidepressant response. J Neuroimmune Pharmacol 2016.

[22] Benedetti F, Riccaboni R, Locatelli C, Poletti S, Dallaspezia S, Colombo C. Rapid treatment response of suicidal symptoms to lithium, sleep deprivation, and light therapy (chronotherapeutics) in drug-resistant bipolar depression. J Clin Psychiatry 2014;75:133-40.

[23] Virtanen J, Ruohonen J, Naatanen R, Ilmoniemi RJ. Instrumentation for the measurement of electric brain responses to transcranial magnetic stimulation. Med Biol Eng Comput 1999;37:322-6.

[24] Casali AG, Casarotto S, Rosanova M, Mariotti M, Massimini M. General indices to characterize the electrical response of the cerebral cortex to TMS. Neuroimage 2010;49:1459-68.

[25] Horne JA. Human sleep, sleep loss and behaviour. Implications for the prefrontal cortex and psychiatric disorder. Br J Psychiatry 1993;162:413-9.

[26] Casarotto S, Romero Lauro LJ, Bellina V, Casali AG, Rosanova M, Pigorini A, et al. EEG responses to TMS are sensitive to changes in the perturbation parameters and repeatable over time. Plos One 2010;5:e10281.

[27] Massimini M, Ferrarelli F, Huber R, Esser SK, Singh H, Tononi G. Breakdown of cortical effective connectivity during sleep. Science 2005;309:2228-32.

[28] Delorme A, Makeig S. EEGLAB: an open source toolbox for analysis of singletrial EEG dynamics including independent component analysis. J Neurosci Methods 2004:134:9-21.

[29] McCulloch CE, Searle SR, Neuhaus JM. Generalized, linear, and mixed models, 2nd ed., New York: John Wiley \& Sons; 2008.

[30] Timm N, Kim K. Univariate and multivariate general linear models: theory and applications with SAS, 2nd ed., Berlin: Heidelberg Springer; 2006.
[31] Hill T, Lewicki P. Statistics: methods and applications. A comprehensive reference for science, industry, and data mining. General Linear Models, StatSoft, Tulsa (OK). 2006; Chapter 18:245-76.

[32] Bartos M, Vida I, Jonas P. Synaptic mechanisms of synchronized gamma oscillations in inhibitory interneuron networks. Nat Rev Neurosci 2007; $8: 45-56$.

[33] Uhlhaas PJ, Haenschel C, Nikolic D, Singer W. The role of oscillations and synchrony in cortical networks and their putative relevance for the pathophysiology of schizophrenia. Schizophr Bull 2008;34:927-43.

[34] Sohal VS, Zhang F, Yizhar O, Deisseroth K. Parvalbumin neurons and gamma rhythms enhance cortical circuit performance. Nature 2009;459:698-702.

[35] Benes FM, Berretta S. Gabaergic interneurons. implications for understand ing schizophrenia and bipolar disorder. Neuropsychopharmacology 2001; 25:1-27.

[36] Whittington MA, Cunningham MO, LeBeau FE, Racca C, Traub RD. Multiple origins of the cortical gamma rhythm. Dev Neurobiol 2011;71:92-106.

[37] Gray CM, McCormick DA. Chattering cells: superficial pyramidal neurons contributing to the generation of synchronous oscillations in the visual cortex. Science 1996;274:109-13.

[38] Traub RD, Bibbig A, LeBeau FE, Cunningham MO, Whittington MA. Persistent gamma oscillations in superficial layers of rat auditory neocortex: experiment and model. J Physiol 2005;562:3-8.

[39] Gonzalez-Burgos G, Lewis DA. GABA neurons and the mechanisms of network oscillations: implications for understanding cortical dysfunction in schizophrenia. Schizophr Bull 2008;34:944-61.

[40] Brenner CA, Kieffaber PD, Clementz BA, Johannesen JK, Shekhar A, O’Donnell $\mathrm{BF}$, et al. Event-related potential abnormalities in schizophrenia: a failure to "gate in" salient information? Schizophr Res 2009;113:332-8.

[41] Wang AY, Lohmann KM, Yang CK, Zimmerman EI, Pantazopoulos H, Herring N, et al. Bipolar disorder type 1 and schizophrenia are accompanied by decreased density of parvalbumin- and somatostatin-positive interneurons in the parahippocampal region. Acta Neuropathol 2011;122:615-26.

[42] Sibille E, Morris HM, Kota RS, Lewis DA. GABA-related transcripts in the dorsolateral prefrontal cortex in mood disorders. Int J Neuropsychopharmacol 2011;14:721-34.

[43] Bhagwagar Z, Wylezinska M, Jezzard P, Evans J, Ashworth F, Sule A, et al. Reduction in occipital cortex gamma-aminobutyric acid concentrations in medication-free recovered unipolar depressed and bipolar subjects. Biol Psychiatry 2007;61:806-12.

[44] Uhlhaas PJ, Singer W. High-frequency oscillations and the neurobiology of schizophrenia. Dialogues Clin Neurosci 2013;15:301-13.

[45] Chitty KM, Lagopoulos J, Lee RS, Hickie IB, Hermens DF. A systematic review and meta-analysis of proton magnetic resonance spectroscopy and mismatch negativity in bipolar disorder. Eur Neuropsychopharmacol 2013;23:1348-63.

[46] Lan MJ, McLoughlin GA, Griffin JL, Tsang TM, Huang JT, Yuan P, et al. Metabonomic analysis identifies molecular changes associated with the pathophysiology and drug treatment of bipolar disorder. Mol Psychiatry 2009;14:269-79.

[47] Korotkova T, Fuchs EC, Ponomarenko A, von Engelhardt J, Monyer H. NMDA receptor ablation on parvalbumin-positive interneurons impairs hippocampal synchrony, spatial representations, and working memory. Neuron 2010. 68:557-69.

[48] Hong LE, Summerfelt A, Buchanan RW, O'Donnell P, Thaker GK, Weiler MA, et al. Gamma and delta neural oscillations and association with clinical symptoms under subanesthetic ketamine. Neuropsychopharmacology 2010;35: 632-40.

[49] Hakami T, Jones NC, Tolmacheva EA, Gaudias J, Chaumont J, Salzberg M, et al. NMDA receptor hypofunction leads to generalized and persistent aberrant gamma oscillations independent of hyperlocomotion and the state of consciousness. Plos One 2009;4:e6755.

[50] Homayoun H, Moghaddam B. NMDA receptor hypofunction produces opposite effects on prefrontal cortex interneurons and pyramidal neurons. J Neurosci 2007;27:11496-500.

[51] Spencer KM. The functional consequences of cortical circuit abnormalities on gamma oscillations in schizophrenia: insights from computational modeling. Front Hum Neurosci 2009;3:33.

[52] Yizhar O, Fenno LE, Davidson TJ, Mogri M, Deisseroth K. Optogenetics in neural systems. Neuron 2011;71:9-34.

[53] Zarate Jr CA, Brutsche NE, Ibrahim L, Franco-Chaves J, Diazgranados N, Cravchik A, et al. Replication of ketamine's antidepressant efficacy in bipolar depression: a randomized controlled add-on trial. Biol Psychiatry 2012; 71:939-46.

[54] Ozerdema A, Guntekind B, Atagune MI, Basar E. Brain oscillations in bipolar disorder in search of new biomarkers. Suppl Clin Neurophysiol 2013;62: 207-21.

[55] Whittington MA, Faulkner HJ, Doheny HC, Traub RD. Neuronal fast oscillations as a target site for psychoactive drugs. Pharmacol Ther 2000;86:171-90.

[56] O'Gorman RL, Poil SS, Brandeis D, Klaver P, Bollmann S, Ghisleni C, et al. Coupling between resting cerebral perfusion and EEG. Brain Topogr 2013;26:442-57

[57] Varela F, Lachaux JP, Rodriguez E, Martinerie J. The brainweb: phase synchronization and large-scale integration. Nat Rev Neurosci 2001;2:229-39.

[58] Lewis AG, Wang L, Bastiaansen M. Fast oscillatory dynamics during language comprehension: unification versus maintenance and prediction? Brain Lang 2015;148:51-63. 
[59] Benasich AA, Gou Z, Choudhury N, Harris KD. Early cognitive and language skills are linked to resting frontal gamma power across the first 3 years. Behav Brain Res 2008;195:215-22.

[60] Kucewicz MT, Cimbalnik J, Matsumoto JY, Brinkmann BH, Bower MR, Vasoli V, et al. High frequency oscillations are associated with cognitive processing in human recognition memory. Brain 2014;137:2231-44.
[61] Benedetti F, Bollettini I. Recent findings on the role of white matter pathology in bipolar disorder. Harv Rev Psychiatry 2014;22:338-41.

[62] Basar E, Schurmann M, Basar-Eroglu C, Demiralp T. Selectively distributed gamma band system of the brain. Int J Psychophysiol 2001; 39:129-35. 